\title{
Wellens' Syndrome, a case report of an electrocardiographic warning sign
}

Yub Raj Sedhai ${ }^{1 *}$, Aditya Singh Pawar ${ }^{1}$, Priyanka T. Bhattacharya ${ }^{2}$, Soney Basnyat ${ }^{3}$

${ }^{1}$ Resident, Department of internal medicine, Mercy Catholic Medical Center, Philadelphia, USA

${ }^{2}$ Hospitalist, Department of internal medicine, Mercy Catholic Medical Center, Philadelphia, USA ${ }^{3}$ Medical Officer, Department of Medicine, Nepal Medical College, Kathmandu, Nepal

\section{DOI Name}

http://dx.doi.org/10.3126/jaim.v5i1.17067

\section{Keywords}

Wellens' Syndrome, Electrocardiogram (ECG),

Coronary Artery disease (CAD), Myocardial

infarction, Takotsubo Cardiomyopathy.

\section{Citation}

Yub Raj Sedhai, Aditya Singh Pawar, Priyandka T. Bhattacharya, et al. Wellens' sydnrome, a case report of an electrographic warning sign..Journal of Advances in Internal Medicine 2016;05(01):19-23.

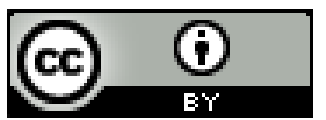

This work is licensed under a Creative Commons Attribution 3.0 Unported License.

\begin{abstract}
\section{Introduction}

Wellens' syndrome is described as a characteristic biphasic or symmetrical electrocardiographic T-wave inversions in precordial leads seen in a subset of patients with unstable angina. It is associated with critical stenosis of left anterior descending coronary artery. These patients have a high likelihood of progressing to acute myocardial infarction within a few days to weeks of onset of symptoms, thus it warrants an early invasive approach.
\end{abstract}

\section{Case Presentation:}

We present a 33-year-old otherwise healthy Indo-Jamaican male who presented with chest pain and characteristic electrocardiographic changes. Wellens' syndrome was recognized. Emergent coronary angiography revealed $99 \%$ stenosis of proximal left anterior descending coronary artery. It was treated with a drug eluting stent.

\section{Conclusion:}

Electrocardiographic changes in Wellens' syndrome are subtle and characteristically appear during chest pain free interval. It can be easily overlooked as non-specific ST, T wave change. Clinicians should be well aware of this subtle yet alarming electrocardiographic sign. This case report underlines the importance of recognizing the ominous electrocardiographic sign and its association with critical stenosis of the left anterior descending coronary artery.

\section{INTRODUCTION/BACKGROUND}

Wellens' syndrome is a subtle electrocardiographic (ECG) manifestation of critical left anterior descending (LAD) coronary artery stenosis. It includes biphasic or deeply inverted $T$ waves in precordial leads. It is seen in a subset of patients with unstable angina during chest pain-free period. This is a pre-infarction condition of coronary artery disease with a high likelihood of developing acute myocardial infarction within a few days to weeks when unintervened ${ }^{1,2}$. We present a case of a young individual with low risk for coronary artery disease. We have discussed the importance of recognizing ECG changes and its association with critical LAD stenosis.

\section{CASE PRESENTATION}

A 33-year-old otherwise healthy Indo-Jamaican male presented to emergency department with sub sternal chest pain for 5 hours. The pain was sudden in onset, aggravated with exercise and was associated with malaise and diaphoresis. He denied having any similar episodes of chest pain in the past. He had a 7-pack-year history of smoking and denied illicit drug and cocaine use. He had no history of diabetes, hypertension,

\footnotetext{
* Corresponding author

Yub Raj Sedhai, MD, Internal Medicine Resident

Mercy Catholic Medical Center, 504, S Lansdowne Avenue, Apt D9

Yeadon, PA, USA, 19050.

dr.sedhai@gmail.com
} 
hyperlipidemia and any history of heart disease in the family. Vital signs on presentation were a blood pressure of 112/68 $\mathrm{mmHg}$, heart rate $88 / \mathrm{min}$, respiratory rate $18 / \mathrm{min}$, temperature $98{ }^{\circ} \mathrm{F}$, and oxygen saturation of $99 \%$ on room air. Body mass index was $33 \mathrm{Kg} / \mathrm{m} 2$. Physical examination of cardiovascular, respiratory and other systems was unremarkable.

\section{Investigations}

Initial electrocardiography (ECG) obtained during pain-free interval revealed T-wave inversion in lead V1 along with biphasic T-waves in septal leads (V2, V3). There was normal progression of R-wave in chest leads, ST segment was isoelectric in the absence pathological Q-waves. This pattern is typical of Wellens' syndrome (Figure-1).

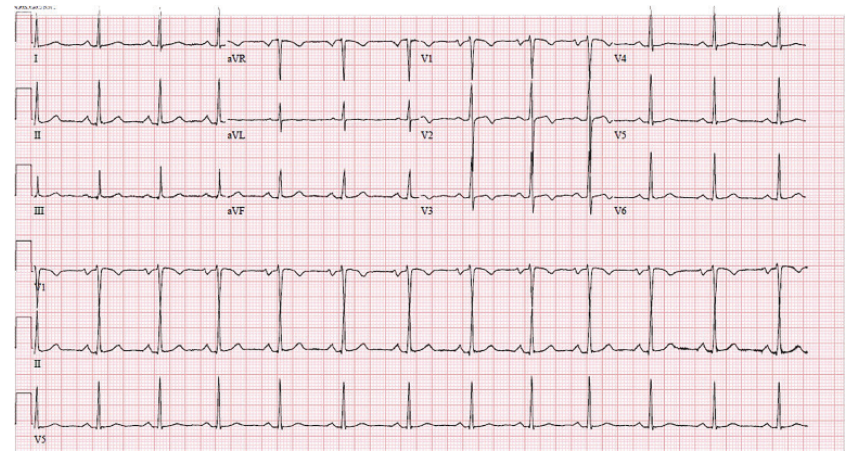

Figure 1: ECG during pain-free interval showing biphasic T-waves in lead V2, V3.

A repeat ECG obtained during chest pain revealed normal of T-waves in lead V2, V3 (Figure 2).

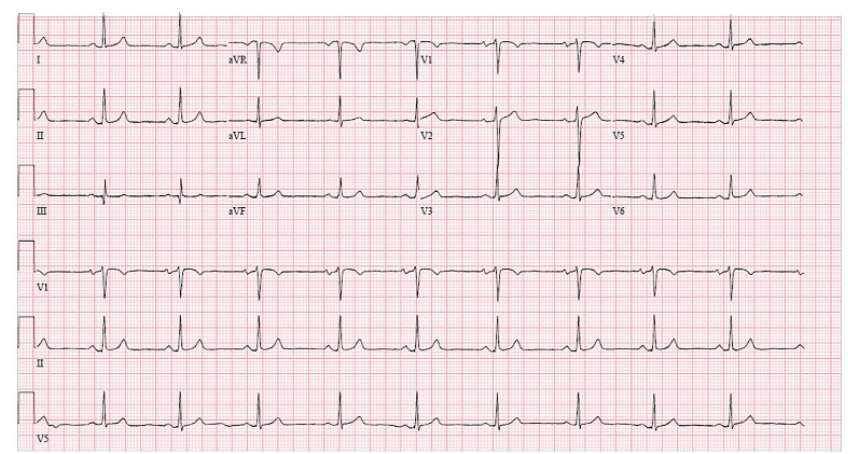

Figure 2: ECG during angina showing resolution of T-wave changes (Pseudo-normalization).

Initial cardiac enzymes were negative, Troponin- $T(<0.01 \mathrm{ng} / \mathrm{ml})$, CK-MB $2 \mathrm{ng} / \mathrm{ml}$. Total cholesterol was $175 \mathrm{mg} / \mathrm{dl}$ and LDL was $135 \mathrm{mg} / \mathrm{dl}$. Random blood glucose was $110 \mathrm{mg} / \mathrm{dl}$ and serum creatinine was $0.6 \mathrm{mg} / \mathrm{dl}$. A bedside 2D-echocardiography showed normal left ventricular chamber size and function. Left ventricular ejection fraction measured $60 \%$ in absence of regional wall motion abnormality. Evaluation of valves and pericardium was unremarkable.

\section{Differential Diagnosis}

Our patient was relatively young, did not have significant risk factors atherosclerotic coronary artery disease and risk stratification by clinical risk scoring tools suggested low risk. TIMI score was 1, GRACE score was 43 and HEART score was 0 . Thus, we initially considered wide range differentials including musculoskeletal chest pain, acute myocarditis, acute pericarditis, pleurisy, pulmonary embolism and TakoTsubo cardiomyopathy (TTM). He did not have any recent history of physical or emotional stress; echocardiography did not reveal apical hypokinesia and ballooning and possibility of TTM became less likely. Presence biphasic T-waves in leads V2, V3 during pain free interval raised our suspicion for Wellens' syndrome. In absence of pronouncing atherosclerotic risk factors we considered the possibility of drug induced coronary vasospasm and performed urinary drug screening. It was negative for cocaine, phencyclidine, marijuana, benzodiazepines and opiates.

\section{Treatment}

With high index of suspicion for Wellens' syndrome, we initially treated our patient by a protocol driven approach for acute coronary syndrome with loading dose of aspirin and unfractionated heparin infusion. Emergent coronary angiography was performed; it revealed $99 \%$ stenosis of the proximal left anterior descending artery distal to diagonal branch 1 in segment B (Figure 3).

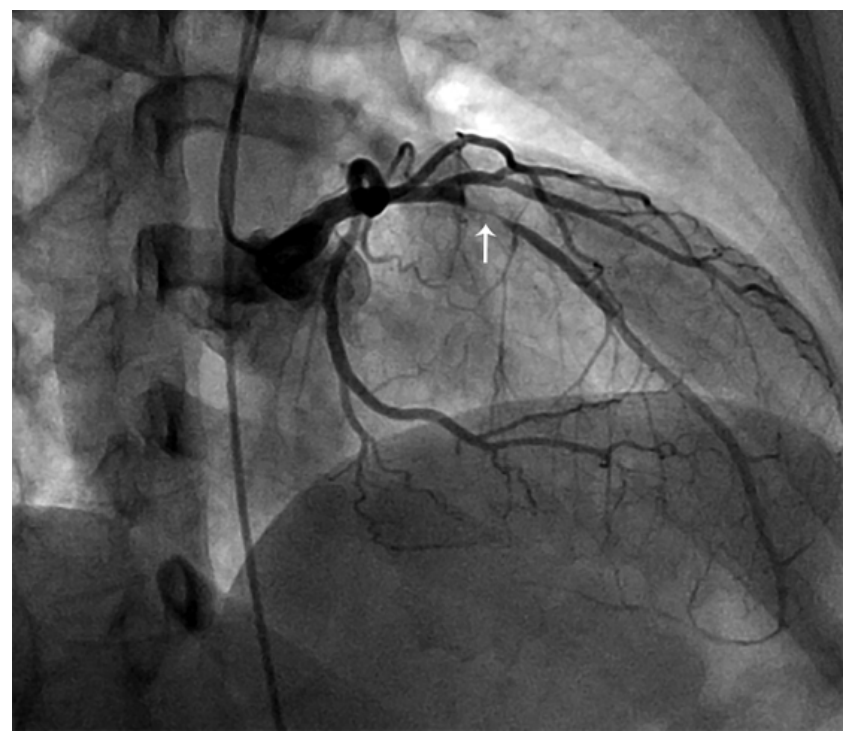

Figure 3: Coronary angiogram showing critical mid left anterior descending artery stenosis (arrow).

It was successfully treated with a drug-eluting stent (Figure 4). After stenting, he was started on dual-anti platelet therapy along with risk stratification medications including ACE-inhibitor, beta-blocker and statin. He had an uneventful 
recovery after revascularization. At discharge, he was enrolled in a smoking cessation and cardiac rehabilitation program.

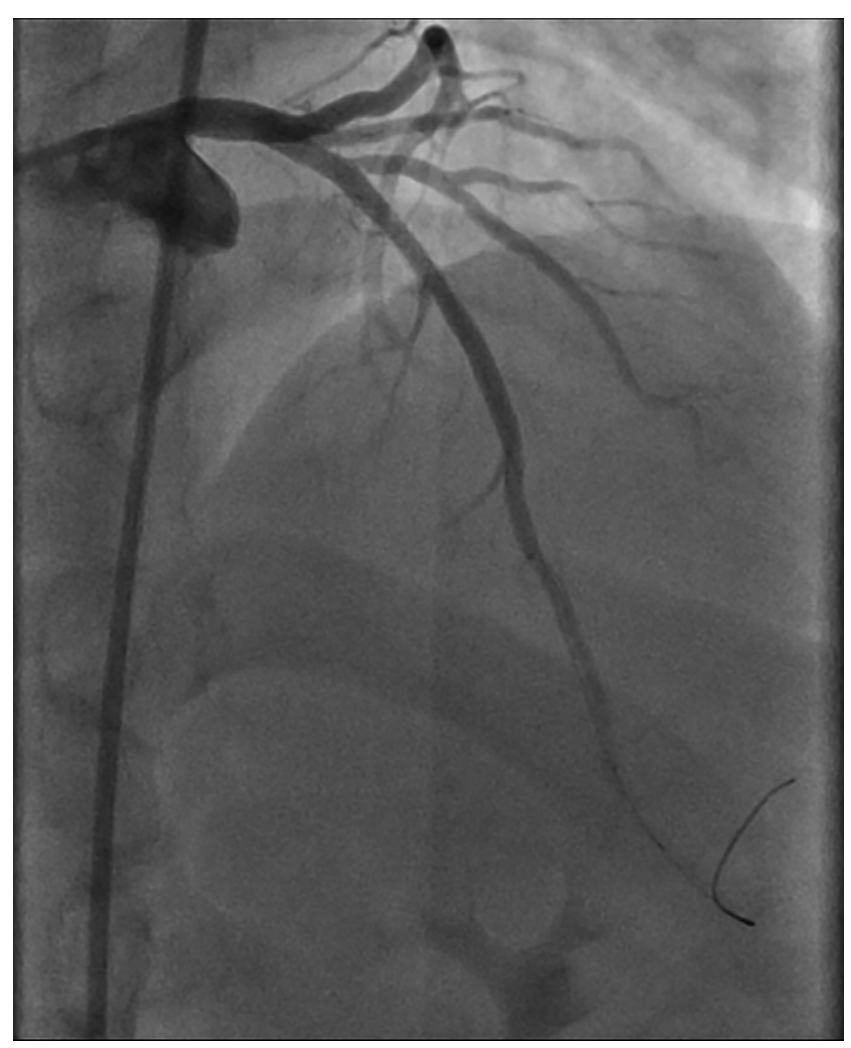

Figure 4: Critical LAD stenosis treated with drug eluting stent.

\section{Outcome and Follow up}

He was followed up at cardiology clinic after 4 weeks. He reported to be symptom-free with an improved effort tolerance.

\section{DISCUSSION}

De Zwann et al. first described Wellens' syndrome as a characteristic pattern of T-wave changes in precordial leads in a subset of patients with unstable angina. It was seen associated with $>90 \%$ stenosis of proximal left anterior descending artery (LAD) and $75 \%$ of patients developed anterior wall myocardial infarction within a few days to weeks ${ }^{1,2,3}$.

There are two distinct morphologic subtypes of Wellens' syndrome.

The common type, Type A comprises $76 \%$ of patients4. Type $A$ is characterized by symmetrical T-wave inversions in V2, V3, often in V4, V5 and sometimes in V6 ${ }^{4}$. Type B is less common and is seen in about $24 \%$ of the patients ${ }^{4}$. Type $B$ is characterized by biphasic T-waves in V2, V3. Type B has a reported association with a higher mortality ${ }^{4}$.

Wellens' syndrome as originally described by De Zwann et al. was associated with critical stenosis of proximal LAD. Location of the lesion in LAD may determine ECG changes. A proximal LAD stenosis jeopardizes a larger territory of myocardium and it may produce more widespread T-wave changes in precordial leads. While, a mid-LAD lesion involves a relatively smaller region of myocardium and ECG change is limited to leads V2, $\mathrm{V}^{6,7}$.

We have mentioned about the potential of Wellens' syndrome to progress to an anterior wall myocardial infarction. It is important to note that Wellens' ECG change is not uncommon. In the initial study by De Zwann et al. 26 out of 145 patients admitted with unstable angina (18\%) had the Wellens' electrocardiographic pattern ${ }^{1}$. In a later prospective study 180 out of 1260 patients (14\%) had the similar ECG pattern. In the first study 12 out of 16 patients (75\%) who were unitervened developed anterior wall myocardial infarction within a few weeks after admission. In the second study all the 180 patients were subjected to coronary angiography and all of them had LAD stenosis ranging from $50 \%$ to complete occlusion ${ }^{2}$.

Causes of electrocardiographic T-wave inversion in precordial leads can be extensive. It includes life-threatening conditions like coronary ischemia and pulmonary embolism. T-wave inversion is also seen in myocarditis, later stage of pericarditis ${ }^{5}$, myocardial straining following ventricular hypertrophy, bundle branch block, pre-excitation syndrome and digitalis effect. The following criteria by Rhinehardt et al. ${ }^{6}$ may be useful in differentiating Wellens' syndrome from other causes of T-wave inversion in precordial leads.

- History of angina,

- Biphasic or deeply inverted T waves in leads V2 and V3; occasionally in leads V1, V4, V5 and V6,

- No or minimal ST elevation $(<1 \mathrm{~mm})$,

- No or minimal elevation of cardiac enzymes,

- No loss of precordial R-wave progression,

- No pathological precordial Q wave.

Discussing further on differentiating Wellens' ECG changes from other causes of T-wave inversion in precordial leads. Bundle branch block will be characterized by widened QRS duration together with characteristic morphologic features of right or left bundle branch block. Left bundle branch block does not produce T-wave inversion in V1 through V4, though it may be seen in Right bundle branch block. In presence of left ventricular hypertrophy with straining, ECG generally shows prominent voltage and repolarization abnormality is mostly seen in Leads I, aVL, V5 and V6. Digitalis induced ECG changes include T-wave inversions with diffuse ST segment depression. Inverted T-waves in digoxin users have a gradual descending limb with a rapid return to base line. T-wave in Wellens' 
Yub Raj Sedhai, et al. Wellens' sydnrome: an electrocardiographic warning sign | Case Report

syndrome is deeply inverted in Type A and biphasic in Type $B^{4}$. Though incidence of pre-mature atherosclerotic coronary artery disease is rising, it is important to consider nonatherosclerotic causes of acute coronary syndrome (ACS) in young adults. Cocaine is well known to cause coronary vasospasm ${ }^{8}$. Marijuana has been incidentally reported to cause coronary vasospasm ${ }^{9}$. Stress cardiomyopathy (TakoTsubo cardiomyopathy) is another differential diagnosis in young adults who present with a suspected ACS particularly when clinical manifestations and electrocardiographic changes are out of proportion of elevation in cardiac biomarkers ${ }^{13}$. ECG changes in Taka-Tsubo Cardiomyopathy (TTM) may include STsegment elevation, ST-segment depression, QT prolongation and T-wave inversion in anterior wall leads ${ }^{10,11}$. In a study by Obied et al. ( $n=200), 22 \%$ of patients with TTM had isolated only isolated T-wave inversions in the precordial leads ${ }^{10}$. This may mimic Type A Wellens' syndrome. Similarly a recently conducted small study $(n=32)$ by Singh et al. ${ }^{10}$, compared $T$ wave width, T-wave depth, QTc interval and proximal and distal T-wave angles in patients with documented TTM versus patients with Wellens' ECG and documented LAD stenosis. No significant difference was seen in two groups except for more number of leads with T-wave inversion in TTM group. Thus, a small percentage ${ }^{11}$ of TTM patients may mimic Wellens' syndrome electrocardiographically and an echocardiography as part of initial assessment may be helpful in distinguishing between the two. Apical hypokinesia and ballooning in echocardiography will be suggestive TTM ${ }^{12,13,14}$.

ECG changes in Wellens' syndrome are subtle and may be easily overlooked as a nonspecific ST-T wave change in an emergency department setting specially in a young patient like ours. ECG changes characteristically appear during the pain-free interval. This makes it even further challenging for clinicians to timely recognize this ominous sign. ECG obtained during chest pain may show upright T-waves with elevated, depressed or isoelectric ST-segment described as pseudonormalization, (Figure 2). This dynamic nature of ECG change is likely related to stunning and reperfusion of myocardium ${ }^{15}$.

Given the dynamic nature of ECG change, it may be missed in a snapshot ECG recorded at the time of admission. This emphasizes the importance of serial ECGs, symptom triggered ECGs and ECG monitoring in patients admitted for chest pain observation. Furthermore, patients with Wellens' ECG pattern should not be subjected to stress testing as underlying severe LAD stenosis may precipitate a myocardial infarction16. In presence of characteristic Wellens' ECG changes, coronary angiography should not be delayed on verge on perusing an alternative diagnosis.

Since our patient was young and appeared low risk for CAD,
ECG findings could be easily overlooked. However, a decision was made to proceed with emergent cardiac catheterization purely based on Wellens' ECG changes. A grievous outcome was prevented by immediate stenting. In conclusion, Wellens' syndrome is an electrocardiographic warning sign. It is important for clinicians to be well aware of the subtle yet alarming ECG change and its association with critical LAD stenosis. Timely recognition and appropriate intervention may prevent a potentially upsetting myocardial infarction.

\section{CONCLUSION}

Clinicians should be well aware about the subtle yet alarming ECG changes of Wellens' syndrome and its association with critical left anterior descending coronary artery stenosis. It is a pre-infarction stage and may progress to an ill-fated myocardial infarction when unintervened. Thus it requires an early invasive approach with coronary angiography in view to consider revascularization. Patients with Wellens' syndrome should not be subjected to stress test.

\section{CONSENT:}

Written informed consent was obtained from the patient for publication of this case report and any accompanying images.

\section{List of abbreviations}

CAD: Coronary artery disease

MI: Myocardial infarction

ECG: Electrocardiography

LAD: Left anterior descending

TTM: Takotsubo cardiomyopathy

\section{Competing Interest}

The authors declare that they have no competing interests.

\section{ACKNOWLEDGEMENT:}

We extend our gratitude to Dr. Eddy Mizrahi MD and Dr. David J. Addley DO for providing cardiac catheterization images. 


\section{REFERENCES:}

1. de Zwaan C, Bär FW, Wellens HJ. Characteristic electrocardiographic pattern indicating a critical stenosis high in left anterior descending coronary artery in patients admitted because of impending myocardial infarction. Am Heart J. 1982 Apr;103(4 Pt 2):730-6.

2. de Zwaan C, Bär FW, Janssen JH, Cheriex EC, Dassen WR, Brugada P, Penn OC, Wellens HJ. Angiographic and clinical characteristics of patients with unstable angina showing an ECG pattern indicating critical narrowing of the proximal LAD coronary artery. Am Heart J. 1989 Mar;117(3):657-65.

3. Movahed MR. Wellens' syndrome or inverted U-waves? Clin Cardiol. 2008 Mar;31(3):133-

4. Nisbet BC, Zlupko G. Repeat Wellens' syndrome: case report of critical proximal left anterior descending artery restenosis. J Emerg Med. 2010 Sep;39(3):305-8.

5. Marx, R Hockberger, R Walls (Eds.), Rosen's Emergency Medicine-Concepts and Clinical Practice (ed 5)Mosby, Inc, St. Louis, MO (2001).

6. Rhinehardt J, Brady WJ, Perron AD, Mattu A. Electrocardiographic manifestations of Wellens' syndrome. Am J Emerg Med. 2002 Nov;20(7):638-43.

7. Sobnosky S, Kohli R, Bleibel S. Wellen's Syndrome. Int J Cardiol. 2006;3:1.

8. Richards JR, Garber D, Laurin EG, Albertson TE, Derlet RW, Amsterdam EA, Olson KR, Ramoska EA, Lange RA. Treatment of cocaine cardiovascular toxicity: a systematic review. Clin Toxicol (Phila). 2016 Jun;54(5):345-64.

9. Co $M$, Okwuosa $T$, Das A. PSEUDO-WELLEN'S SYNDROME AFTER MARIJUANA USE. J Am Coll Cardiol.
2016;67(13_S):1166.

10. Singh M, Harlapur M, Acharya T, Wessel R, Bhullar A, Ambrose J. T WAVE AND QT CHANGES IN TAKOTSUBO CARDIOMYOPATHY VERSUS WELLENS ' T WAVES. J Am Coll Cardiol. 2016;67(13_S):524.

11. Obeid S, Frangieh AH, Ghadri JR, Lüscher TF, Templin C. CHARACTERISTICS AND SPECIFIC ECG FINDINGS IN 200 PATIENTS WITH STRESS-INDUCED "TAKOTSUBO" CARDIOMYOPATHY COMPARISON BETWEEN TYPICAL AND ATYPICAL FORMS.J Am Coll Cardiol. 2015;65(10_S):.

12. Horowitz JD, Nguyen TH. Role of Echocardiography in Tako-Tsubo Cardiomyopathy: Beyond Diagnosis? J Am Coll Cardiol Img. 2014;7(2):130-132.

13. Amsterdam EA, Wenger NK, Brindis RG, et al. 2014 AHA/ ACC Guideline for the Management of Patients With Non-ST-Elevation Acute Coronary Syndromes: A Report of the American College of Cardiology/American Heart Association Task Force on Practice Guidelines.J Am Coll Cardiol. 2014;64(24):e139-e228.

14. Dec GW. Recognition of the apical ballooning syndrome in the United States. Circulation. 2005 Feb 1;111(4):38890.

15. Migliore F, Zorzi A, Marra MP, Basso C, Corbetti F, De Lazzari M, Tarantini G, Buja P, Lacognata C, Thiene G, Corrado D, Iliceto S. Myocardial edema underlies dynamic T-wave inversion (Wellens' ECG pattern) in patients with reversible left ventricular dysfunction. Heart Rhythm. 2011 Oct;8(10):1629-34.

16. Patel K, Alattar F, Koneru J, Shamoon F. ST-Elevation Myocardial Infarction after Pharmacologic Persantine Stress Test in a Patient with Wellens' Syndrome. Case Rep Emerg Med. 2014;2014:530451. 